\title{
Lack of association between ipratropium bromide and mortality in elderly patients with chronic obstructive airway disease
}

\author{
Don D Sin, Jack V Tu
}

\begin{abstract}
Background-Ipratropium is commonly used for the management of elderly patients with obstructive airway disease. However, a recent report suggested that its use might be associated with a significant increase in mortality. A study was therefore conducted to compare all-cause mortality rates between users and nonusers of ipratropium in elderly patients with either asthma or chronic obstructive pulmonary disease (COPD).

Methods-A retrospective cohort study was performed using linked data from the Canadian Institute for Health Information, the Ontario Drug Benefit Program, the Ontario Health Insurance Plan, and the Ontario Registered Persons database. A total of 32393 patients were identified who were aged 65 years or older and who had been discharged from hospital with asthma or COPD between 1 April 1992 and 31 March 1997. All-cause mortality rates were compared between those treated and those not treated with ipratropium following discharge from hospital. Results-In total, $49 \%$ of patients received ipratropium within 90 days of discharge. After adjusting for age, sex, comorbidity, use of health services, and other airway medications there was no significant association in patients with COPD between the use of ipratropium and mortality (relative risk (RR) $1.03 ; 95 \%$ confidence interval (CI) 0.98 to 1.08 ). In patients with asthma, however, there was a slight increase in the relative risk of mortality associated with the use of ipratropium (RR 1.24; 95\% CI 1.11 to 1.39 ). A doseresponse increase in the mortality rate was not observed with increasing use of ipratropium in either COPD or asthma.

Conclusions-The use of ipratropium in patients with COPD was not associated with an increase in mortality. However, in asthma there was a small increase in the mortality rate. Since asthmatic patients who received ipratropium had greater use of other airway medications and health services, the difference in mortality rate between users and non-users may be a reflection of unmeasured differences in asthma severity.

(Thorax 2000;55:194-197)
\end{abstract}

Keywords: ipratropium; mortality; asthma; chronic obstructive pulmonary disease
Obstructive airway disease is a progressive condition that causes a great deal of morbidity and mortality. ${ }^{1}$ Despite recent improvements in its management, mortality rates from obstructive airway disease continue to rise. ${ }^{2}$ There is growing concern that some of the currently available medications are contributing to the increase in morbidity and mortality. ${ }^{3}$ For example, in Canada mortality was shown to be higher in regular users of inhaled $\beta_{2}$ agonists than in infrequent users. ${ }^{4}$ On the other hand, inhaled anticholinergic drugs have been considered safe and effective for the management of both asthma and chronic obstructive pulmonary disease (COPD)..$^{5}$ Although these medications have not been shown to change the natural course of the disease, they enhance lung function, patient symptoms, and quality of life for those with significant airflow limitation. ${ }^{6}$ Indeed, various international consensus guidelines have recommended that inhaled anticholinergic drugs should be used as front line agents in the treatment of COPD and as a second line agent for asthma. ${ }^{7}$ The long term safety of these drugs, however, has not been well studied.

A recent report suggested that these agents may not be safe for use in those who have previously been admitted to hospital with asthma. ${ }^{9}$ In fact, use of these drugs following discharge from hospital was associated with a large increase in mortality. However, since asthmatic patients treated with inhaled anticholinergic drugs are more likely to have a co-existing component of fixed airway disease, and since these patients have a prognosis that is different from those with uncomplicated asthma, ${ }^{10}$ these results may have been confounded by unmeasured differences in asthma severity between users and non-users of these drugs.

In view of the widespread use of inhaled anticholinergics for the management of COPD and asthma in the community, ${ }^{10}$ we sought to determine whether the use of inhaled anticholinergic drugs was associated with an increased risk of all-cause mortality among elderly patients with obstructive airway disease. We conducted a population based study of patients with asthma and COPD and evaluated the effect of inhaled anticholinergic therapy separately for each of these groups. We reasoned that, if inhaled anticholinergics increased mortality, the effect would be similar in both asthmatic and COPD patients. On the other hand, if the use of inhaled anticholinergics was just a marker of disease severity, the risk of mortality may be spuriously increased in 
asthma but not in COPD since these drugs are generally used as first line agents in COPD but not in asthma.

\section{Methods}

STUDY POPULATION

The Canadian Institute for Health Information (CIHI) hospital discharge database was used to identify 32393 consecutive individuals aged 65-99 years in Ontario, Canada who had been admitted to hospital at least once with a most responsible diagnosis of COPD or asthma between 1 April 1992 and 31 March 1997. We restricted our study to those aged 65 years and older because outpatient drug information in the younger population was not available to us. These patients were categorised to either the asthma or COPD group based on the International Classification of Diseases, 9 th revision (ICD-9) diagnostic codes. ICD-9 codes 490, 491, 492, 496 were used for COPD and ICD-9 code 493 for asthma. The accuracy and validity of these ICD-9 codes in the CIHI database have been demonstrated previously. ${ }^{11}$ The index hospital admission and discharge was therefore defined as the date of the first admission and discharge from hospital during the study period, respectively.

EXPOSURE TO IPRATROPIUM AND POTENTIAL CONFOUNDERS

The use of ipratropium bromide was identified from the Ontario Drug Benefit (ODB) database. The ODB programme provides free outpatient medications to Ontario residents aged 65 years or over. "Initial users" of ipratropium were defined as subjects who were issued with at least one prescription for the drug within 90 days of the index discharge date.

Disease severity was estimated for each patient using the following surrogate markers: (1) dispensing of other airway medications within 90 days of discharge-these included inhaled $\beta_{2}$ agonists (fenoterol, orciprenaline, salbutamol, terbutaline sulfate), inhaled corticosteroids (beclomethasone dipropionate, budesonide, fluticasone propionate, triamcinolone acetate), oral corticosteroids, and oral theophylline derivatives; (2) use of health services (emergency and outpatient physician visits for COPD or asthma) during the year before the index hospital admission from the Ontario Health Insurance Plan (OHIP) physician claims database; and (3) length of stay during the index admission to hospital from the CIHI database. In addition, for each patient secondary diagnoses in the CIHI database were used to construct a Charlson comorbidity index score $^{12}$ which was used to control for comorbidity. We did not assign any points for COPD in calculating these scores because it was one of our primary diagnoses. Age and sex were also considered potential confounders.

OUTCOMES

Vital status information on all patients was obtained through the Registered Persons database of Ontario which contains a computerised list of all people who die in Ontario, including the date of death. This database is considered accurate and comprehensive since, by provincial law, all deaths occurring in Ontario must be reported. The accuracy and validity of these databases have been demonstrated previously. ${ }^{13}$ We could not determine the cause of death for our patients since this information was not captured in this database. Thus, we restricted our analysis to all-cause mortality.

For the initial analysis the patients were followed from the date of discharge from the index hospital admission to the date of their death, one year following their discharge date, or the end of the follow up period (31 March 1998), whichever came first. This latter date was selected in order to ensure that all surviving patients in the cohort had at least one year of potential follow up. In the secondary analysis a dose-response relationship between ipratropium use and mortality rate was evaluated. For this analysis the end of the follow up time was slightly modified; the patients were followed from the date of discharge to the date of their death or 31 March 1998, whichever came first.

STATISTICAL ANALYSIS

Wilcoxon rank sum and $t$ tests were used to compare non-parametric and parametric continuous variables, respectively; $\chi^{2}$ tests were used for dichotomous variables. Mortality was compared between those treated and those not treated with ipratropium by the Cox proportional hazards model. In the primary analysis the exposure variable of interest was whether or not patients were dispensed with at least one prescription of ipratropium in the first 90 days following discharge from the index hospital admission - that is, "initial users". We treated age as a continuous variable and the Charlson score as ordered categorical data $(0,1,2, \geqslant 3)$. All other variables, including administration of airway medications such as inhaled steroids, $\beta_{2}$ agonists, and oral steroids, were treated as dichotomous variables. Adjusted analysis was conducted to determine whether the dosing frequency of ipratropium was related to mortality. For each patient we obtained the total number of prescriptions of ipratropium that were filled during the first two years of the follow up period. Patients were then grouped according to the number of prescriptions dispensed and the rate of mortality was calculated, adjusting for person-years of follow up. Those who did not fill any prescription for ipratropium were used as the reference group. Comparisons between the reference group and other dispensing categories were made using Poisson regression. We also conducted a sensitivity analysis in which patients who died within the first 90 days were excluded from the analysis. This did not significantly change our results. All analyses were conducted using SAS software, release 6.12 (SAS Institute, Cary, NC, USA).

\section{Results}

Over the study period 6589 elderly individuals were discharged from hospital in Ontario for asthma and 25804 for COPD. The amount of observation time was 20785 person-years for 
Table 1 Characteristics of patients with asthma and COPD with and without exposure to ipratropium in the first 90 days following discharge

\begin{tabular}{|c|c|c|c|c|}
\hline & \multicolumn{2}{|l|}{$C O P D$} & \multicolumn{2}{|l|}{ Asthma } \\
\hline & $\begin{array}{l}\text { No ipratropium } \\
(n=12591)\end{array}$ & $\begin{array}{l}\text { Ipratropium } \\
(n=13213)\end{array}$ & $\begin{array}{l}\text { No ipratropium } \\
(n=4012)\end{array}$ & $\begin{array}{l}\text { Ipratropium } \\
(n=2577)\end{array}$ \\
\hline Mean (SD) age (years) & $76.2(7.1)$ & $75.1(6.7)$ & $74.8(7.3)^{\star}$ & $74.6(6.8)^{\star}$ \\
\hline Men & $6852(54.4 \%)$ & $7514(56.9 \%)$ & $1163(29.0 \%)$ & $889(34.5 \%)$ \\
\hline Charlson index $\geqslant 1$ & $5634(44.8 \%)$ & $5715(43.3 \%)$ & $1634(40.7 \%)$ & $1135(44.0 \%)$ \\
\hline \multicolumn{5}{|c|}{ Mean (SD) no. of health service visits and drug usage during year prior to hospital admission: } \\
\hline Emergency room & $0.02(0.17)$ & $0.05(0.24)$ & $0.05(0.24)$ & $0.07(0.28)$ \\
\hline Physician's office & $1.47(1.79)$ & $2.37(2.14)$ & $1.54(1.80)$ & $2.36(2.11)$ \\
\hline$\beta_{2}$ agonists & $2.27(2.67)$ & $3.72(2.83)$ & $2.35(2.62)$ & $3.63(2.79)$ \\
\hline Inhaled steroids & $1.53(2.39)$ & $2.80(2.93)$ & $1.78(2.38)$ & $3.02(2.82)$ \\
\hline Oral steroids & $0.62(1.56)$ & $1.03(1.89)$ & $0.78(1.59)$ & $1.19(1.91)$ \\
\hline Oral theophyllines & $0.71(1.96)$ & $1.17(2.42)$ & $0.60(1.75)$ & $0.94(2.12)$ \\
\hline \multicolumn{5}{|c|}{ Receipt of $\geqslant 1$ prescription for other airway medications during 90 days after discharge: } \\
\hline$\beta_{2}$ agonists & $5270(41.9 \%)$ & $12227(92.5 \%)$ & $2082(51.9 \%)$ & $2342(90.9 \%)$ \\
\hline Inhaled steroids & $3992(31.7 \%)$ & $8612(65.2 \%)$ & $1912(47.7 \%)$ & $2033(78.9 \%)$ \\
\hline Oral steroids & $2325(18.5 \%)$ & $5316(40.2 \%)$ & $1135(28.3 \%)$ & $1279(49.6 \%)$ \\
\hline Oral theophylline & $1387(11.0 \%)$ & $3191(24.2 \%)$ & $471(11.7 \%)$ & $523(20.3 \%)$ \\
\hline
\end{tabular}

All comparisons between users and non-users of ipratropium in COPD and asthma groups are significant at $\mathrm{p}<0.001$ except where indicated. ${ }^{\star} \mathrm{p}=0.352$.

asthma and 60661 person-years for COPD. The overall median duration of observation time was three years for both groups. Of these 32393 patients, 15790 (49\%) were dispensed at least one prescription of ipratropium within 90 days of the index discharge date. Only 2577 $(39 \%)$ asthmatics received at least one prescription for the drug during this time interval compared with 13213 (51\%) patients with COPD. Overall, those who received ipratropium were slightly younger and more likely to be men. They were also more likely to receive other airway medications including inhaled $\beta_{2}$ agonists, inhaled and oral steroids, and oral theophyllines and to have higher utilisation rate of outpatient physician and emergency services compared with those without such exposure (table 1).

A total of 16557 deaths was observed, 2072 $(31.5 \%)$ in the asthma group and 14485 $(56.1 \%)$ patients with COPD. In a crude analysis asthmatic patients who were initial users of ipratropium had a $41 \%$ (95\% confidence interval (CI) 30 to 54) higher relative risk of all-cause mortality than those who were initial non-users. In the COPD group users of ipratropium had only a $7 \%(95 \%$ CI 3 to 10$)$ increased risk of mortality. After adjustments for disease severity, age, sex and comorbidity, the relative risk was decreased substantially for both conditions. In patients with asthma there

Table 2 Risk of long term mortality in elderly patients, stratified according to the number of prescriptions of ipratropium bromide dispensed during the study period

\begin{tabular}{llll}
\hline $\begin{array}{l}\text { No. of prescriptions } \\
\text { dispensed }\end{array}$ & No. of patients & $\begin{array}{l}\text { Mortality rate } \\
\text { (per 100 person-years) }\end{array}$ & $\begin{array}{l}\text { Relative risk for } \\
\text { mortality }\end{array}$ \\
\hline $\begin{array}{c}\text { Asthma } \\
0\end{array}$ & 2733 & 8.35 & 1.0 (reference) \\
$1-2$ & 1409 & 10.08 & $1.14(0.99$ to 1.31$)$ \\
$3-4$ & 763 & 12.37 & $1.17(0.98$ to 1.40$)$ \\
$5-6$ & 414 & 12.73 & $1.26(1.06$ to 1.50$)$ \\
$7-8$ & 964 & 10.99 & $1.26(1.06$ to 1.51$)$ \\
$\geqslant 9$ & 306 & 7.71 & $0.81(0.61$ to 1.08$)$ \\
COPD & & & 1.0 (reference) \\
0 & 7832 & 25.00 & $1.20(1.13$ to 1.27$)$ \\
$1-2$ & 4952 & 32.73 & $1.07(1.00$ to 1.15$)$ \\
$3-4$ & 3426 & 28.87 & $1.02(0.94$ to 1.09$)$ \\
$5-6$ & 4074 & 23.58 & $0.91(0.85$ to 0.98$)$ \\
$7-8$ & 4319 & 17.12 & $0.85(0.76$ to 0.94$)$ \\
$\geqslant 9$ & 1201 & 13.96 & \\
\hline
\end{tabular}

*Ajusted for age, sex, comorbidity, length of stay, use of other airway medications and health services (emergency department and physician visits). was a $24 \%$ (95\% CI 11 to 39$)$ increase in the adjusted risk while in those with COPD the increase in adjusted risk was only 3\% (95\% CI -2 to 8 ) which was not statistically significant $(\mathrm{p}=0.119)$.

An analysis which took into account differences in the number of prescriptions of ipratropium issued during the follow up period did not show a dose-response relationship between the use of ipratropium and mortality in either the asthma or COPD group of patients (table 2). Although there was a slight increase in the relative risk of mortality among elderly asthmatics, in four of the six dispensing categories the lower limit of the $95 \%$ CI was $\leqslant 1.0$ which indicated no significant increase in risk. In patients with COPD this was true in five of the six dispensing categories.

\section{Discussion}

We did not observe a significant or consistent association between the use of ipratropium bromide and all-cause mortality in elderly patients with COPD. In a crude analysis the relative risk of mortality was slightly increased among initial users of ipratropium, but adjustments for age, sex, comorbidity, and severity of disease-as measured by various surrogate markers-markedly reduced the effect size. Indeed, adjustments for these confounders in patients with COPD resulted in no significant association, while in patients with asthma the use of ipratropium was associated with only a marginal increase in the risk of mortality. Moreover, we did not observe a dose-response increase in mortality associated with increasing use of ipratropium in either asthma or COPD.

The weak association in asthma can probably be explained by residual confounding by severity. Since ipratropium is regarded as a second line agent in asthma, those who received it are likely to have had more severe asthma than non-users. ${ }^{8}$ Indeed, among users of ipratropium there was a higher frequency of visits to the emergency room and outpatient physician offices for asthma and a greater use of all other anti-asthma medications prior to the initial hospital admission. Moreover, since ipratro- 
pium appears to be more effective in COPD than in asthma, asthmatics who received this drug may have had a significant coexisting component of fixed airway disease which may have worsened their prognosis. ${ }^{10}$ On the other hand, since ipratropium is a front line agent in COPD, residual confounding by severity was less likely to have been present. No significant association between exposure to ipratropium and mortality, after adjustments for various confounders, was seen in patients with COPD, which suggests that exposure to ipratropium does not increase the risk of mortality in obstructive airway disease. An alternative explanation, however, is that the risk of mortality with the use of ipratropium is modified by the presence of asthma. However, there is no strong physiological reason for this to be the case.

The relatively low usage of inhaled steroids and $\beta_{2}$ agonists in those not treated with inhaled anticholinergic agents after discharge raises the possibility that some of these patients may not have had COPD or asthma as their primary condition. However, previous validation of the CIHI database suggests that the specificity and sensitivity of using the most responsible diagnosis for obstructive airway disease are accurate and reliable. ${ }^{11}$ Thus, diagnostic misclassification is unlikely to account for our observations. The lower usage of these medications in this group may reflect a lower degree of disease severity compared with those treated with inhaled anticholinergics after discharge.

Our results differ from those of Guite et al who showed that initial use of ipratropium was associated with a 4.04-fold increase in asthma mortality and a 7.75-fold increase in COPD mortality. ${ }^{9}$ However, in contrast to our study, their cohort comprised only asthmatic patients. Since asthmatic patients who use ipratropium are likely to have more severe asthma than non-users, their results may have been confounded by indication. Furthermore, their results may have been limited by the small number of deaths observed during the study period, producing unstable point estimates. Our patients had more severe asthma and we observed over 2000 deaths in the asthma cohort, making our results much more stable. Another strength of our study was that it was population based so the results should therefore be more applicable to the general population of elderly asthmatic and COPD patients than those of previous studies. Since we did not have information on cause of death, we cannot rule out the possibility that ipratropium may selectively increase the risk for certain causes of death. It is reassuring, however, that we did not observe an increase in the overall all-cause mortality rate with use of ipratropium.

Our results are consistent with the pharmacokinetic properties of ipratropium. Systemic side effects from ipratropium are rare since less than $1 \%$ of the drug is absorbed into the systemic circulation. ${ }^{5}$ Moreover, even in larger than normal doses, ipratropium has not been consistently shown to decrease mucociliary clearance or to affect the viscoelastic properties of tracheal mucus ${ }^{5}$; thus, long term use of ipratropium is likely to be safe. Indeed, long term studies of ipratropium have not yielded higher mortality rates in those treated with the drug. ${ }^{14} 15$

In summary, the results of our study suggest that the use of ipratropium bromide is not associated with an increased risk of all-cause mortality in patients with COPD. The slight excess risk observed in elderly asthmatic subjects probably reflects differences in the severity of asthma between those treated and those not treated with inhaled anticholinergics. We therefore conclude that ipratropium appears to be safe for the treatment of elderly patients with obstructive airway disease.

Dr Sin was supported by a fellowship from the Alberta Heritage Foundation for Medical Research at the time of this work. Dr Tu is supported by a Medical Research Council of Canada Scholarship Award. This work was supported in part by ICES which is funded by the Ontario Ministry of Health. The results and conclusions are strictly those of the authors and should not be conclusions are strictly those of the authors
attributed to any of the sponsoring agencies.

1 Speizer FE. The rise in chronic obstructive pulmonary disease mortality: overview and summary. Am Rev Respir Dis 1989;140(Suppl): 106-7.

2 Feinlieb M, Rosenberg HM, Collins JG, et al. Trends in COPD morbidity and mortality in the United States. $\mathrm{Am}$ Rev Respir Dis 1989;140(Suppl):9-18.

3 Spitzer WO, Suissa S, Ernst P, et al. The use of beta-agonists and the risk of death and near death from asthma. NEngl $\mathcal{F}$ Med 1992;326:501-6.

4 Suissa S, Ernst P, Boivin J, et al. A cohort analysis of excess mortality in asthma and the use of inhaled $\beta$-agonists. $A m \mathcal{F}$ Respir Crit Care Med 1994;149:604-10.

5 Gross NJ. Ipratropium bromide. N Engl f Med 1988;319: 486-94.

6 The Combivent Inhalation Solution Study Group. Routine nebulized ipratropium and albuterol together are better than either alone in COPD. Chest 1997;112:1514-21.

7 The COPD Guidelines Group of the Standards of Care Committee of the British Thoracic Society. BTS guidelines for the management of chronic obstructive pulmonary disease. Thorax 1997;52(Suppl 5):1-28.

8 Expert Panel Report 2. Guidelines for the diagnosis and management of asthma. NHLBI/WHO Workshop report. National Institutes of Health, National Heart, Lung and Blood Institute, Publication No. 95-3659, 1995.

9 Guite HF, Dundas R, Burney PGJ. Risk factors for death from asthma, chronic obstructive pulmonary disease and
from cardiovascular disease after a hospital admission for asthma. Thorax 1999;54:301-7.

10 Meir CR, Jick H. Drug use and pulmonary death rates in increasingly symptomatic asthma patients in the UK. Tho$\operatorname{rax}$ 1997;52:616-7.

11 Rawson NS, Malcolm E. Validity of the recording of ischemic heart disease and chronic obstructive pulmonary disease in the Saskatchewan health care datafiles. Stat Med 1995; 14:2627-43.

12 Deyo RA, Cherkin DC, Ciol MA. Adapting a clinical comorbidity index for use with ICD-9-CM administrative databases. F Clin Epidemiol 1992;45:613-29.

13 Tu JV, Pashos CL, Naylor CD, et al. Use of cardiac procedures and outcomes in elderly patients with myocardial infarction in the United States and Canada. $N$ Engl $\mathcal{F}$ Med 1998;336:500-5.

14 Anthonisen NR, Connett JE, Kiley JP, et al. Effects of smoking intervention and the use of an inhaled anticholinergic bronchodilator on the rate of decline of $\mathrm{FEV}_{1}$. The Lung Health Study. fAMA 1994;272:497-505.

15 Rennard SI, Serby CW, Ghafouri M, et al. Extended therapy with ipratropium is associated with improved lung function in patients with COPD. A retrospective analysis of data from seven clinical trials. Chest 1996;110:62-70. 